\title{
Comercio electrónico como medio de estrategia para el impulso de productos artesanales
}

\section{Electronic commerce as a means of strategy to promote artisan products}

\author{
Valeria Jiménez Cervantes ${ }^{1}$, Dr. Germán Martínez Prats ${ }^{2}$, Dra. Francisca Silva \\ Hernández ${ }^{3}$
}

${ }^{1}$ Universidad Juárez Autónoma de Tabasco, México vcervantes.ro@gmail.com https://orcid.org/0000-0002-3181-8580

²Universidad Juárez Autónoma de Tabasco, México germanmtzprats@hotmail.com

ORCID: https://orcid.org/0000-0001-6371-448X

3Universidad Juárez Autónoma de Tabasco, México fany987@hotmail.com ORCID: https://orcid.org/0000-0003-3533-0002

Dol https://doi.org/10.46589/rdiasf.vi35.372

Recibido 08 de enero 2021.

Aceptado 19 abril 2021

Publicado 30 de junio de 2021

\section{Resumen}

El comercio electrónico garantiza un mejor y amplio desarrollo para el segmento primario, buscando colaborar con el mismo para dar el salto a las nuevas tecnologías logrando así darse a conocer y expandir negocios con un nuevo modelo de mercado. Para ello, es importante considerar estudio de factibilidad de mercado, que permita determinar las opciones para colaborar a la integración de un nuevo modelo de negocio y ajustar las estrategias que ayuden a propiciar el fomento de la difusión cultural y un mayor apoyo para el desarrollo de las pequeñas empresas dedicadas al giro comercial artesanal. De este modo se permite el desarrollo de la actividad artesanal aportando que con este proyecto haya un mayor crecimiento de este segmento de la población y su alcance no sólo es local sino también internacional coadyuvando a la preservación y difusión de las tradiciones culturales de las entidades.

Palabras clave: Comercio electrónico, economía, plataforma digital, producto artesanal 


\begin{abstract}
Electronic commerce guarantees a better and broad development for the primary segment, seeking to collaborate with it to make the leap to new technologies, thus making itself known and expanding business with a new market model. For this, it is important to consider a market feasibility study, which allows determining the options to collaborate in the integration of a new business model and adjusting the strategies that help promote the promotion of cultural diffusion and greater support for the development of small companies engaged in the artisanal business. In this way, the development of artisanal activity is allowed, contributing with this project to a greater growth of this segment of the population and its scope is not only local but also international, contributing to the preservation and dissemination of the cultural traditions of the entities.
\end{abstract}

Key words: Electronic commerce, economy, digital platform, artisan product.

\title{
Introducción
}

Uno de los principales problemas que tiene el sector artesanal es todavía la falta de promoción y seguimiento de programas o iniciativas que impulse un mejor desarrollo dentro de su segmento de mercado, quedando rezagados por las nuevas tecnologías y competencias como marcas reconocidas de moda, que hoy en día crean productos similares que suplen a lo artesanal provocando de esa manera que su valor se deprecie considerablemente a ojos de las generaciones actuales y futuras.

Existen programas auspiciados por el gobierno que propician la ayuda económica para la producción de productos artesanales y la difusión del comercio de las artesanías a través de portales en línea. Lamentablemente la falta de constancia y mantenimiento de los sitios web no permiten el desarrollo adecuado de los involucrados, limitando de esa manera la comercialización de los productos artesanales a un nivel más elevado del mercado nacional.

Otro factor importante es la falta de herramientas tecnológicas y accesos a internet, ya que muchos artesanos no están inscritos a programas de apoyo por parte del gobierno y no pueden permitirse incursionar por cuenta propia a la nueva revolución digital que día a día ofrece nuevas y mejores oportunidades de crecimiento en el mercado.

Es por ello, que por medio de esta investigación se busca poder apoyar a las pequeñas empresas familiares dedicadas al rubro artesanal, a impulsar su negocio (Ochoa et al, 2015) y poder llegar a un nivel más amplio de reconocimiento y venta. De igual manera reafirmar el valor que tienen las artesanías no sólo a un nivel local o estatal sino abarcar el mercado internacional con ayuda de estrategias mercadológicas. 


\section{Cultura y artesanía}

La cultura es una red de relaciones en la cual las artesanías abren una brecha para comprender y visualizar la conexión entre los elementos naturales, sociales y simbólicos del grupo social que las produce. Entendiéndose por cultura a la manera de ver la vida de una comunidad humana, su modo de pensarse a sí mismos, de comunicarse, de construir una sociedad y una serie de valores trascendentes, que pueden ir desde la religión, la moral, las artes, el protocolo, la ley, la historia, la economía etc.

Los productos artesanales son los producidos por artesanos, ya sea totalmente a mano, o con la ayuda de herramientas manuales o incluso de medios mecánicos, siempre que la contribución manual directa del artesano siga siendo el componente más importante del producto acabado (UNESCO, 2017). Se producen sin limitación por lo que se refiere a la cantidad y utilizando materias primas procedentes de recursos sostenibles (Martínez et al, 2020).

Para muchos, las artesanías son un término medio entre el diseño y el arte, Roncancio (1999) define las artesanías son el resultado de la creatividad y la imaginación, plasmado en un producto en cuya elaboración se ha transformado racionalmente materiales de origen natural, generalmente con procesos y técnicas manuales. Los objetos artesanales van cargados de un alto valor cultural y debido a su proceso son piezas únicas.

Es por ese alto valor cultural que la correcta difusión y uso de estrategias mercadológicas en conjunto a la comercialización de productos artesanales a través de internet pueden contribuir a un mejor desarrollo económico y reconocimiento cultural a un nivel más amplio de mercado.

\section{Mercadotecnia digital}

La mercadotecnia se define como "la ciencia y el arte de explorar, crear y entregar valor para satisfacer las necesidades de un mercado objetivo por un beneficio. La mercadotecnia identifica necesidades insatisfechas y deseos. Define, mide y cuantifica el tamaño del mercado y su potencial de ganancias. Señala qué segmentos la compañía sirven mejor y diseña y promueve los productos y servicios adecuados" (Kotler \& Armstrong, 2003: 397).

En Marketing se hace uso de estrategias de mercado para trazar y alcanzar los objetivos que como empresa se propone a criterio una estrategia de mercadotecnia como aquel que "es un tipo de estrategia que define un mercado meta y la combinación de mercadotecnia relacionada con él. En pocas palabras se 
trata de un de panorama general sobre el modo de actuar de una empresa dentro de un mercado." (Jerome \& Perreault, 1996: 127)

Según Laura Fischer y Jorge Espejo (2004) describen que las estrategias de mercadotecnia "comprende la selección y el análisis del mercado, es decir, la elección y el estudio del grupo de personas a las que se desea llegar, así como la creación y permanencia de la mezcla de mercadotecnia que las satisfaga".

\section{Mercadotecnia digital. (Marketing digital)}

El Marketing Digital es la aplicación de estrategias de comercialización llevadas a cabo en los medios digitales. Todas las técnicas del mundo off-line son imitadas y traducidas a un nuevo mundo, el mundo online. En el ámbito digital aparecen nuevas herramientas como la inmediatez, las nuevas redes que surgen día a día, y la posibilidad de mediciones reales de cada una de las estrategias empleadas.

Asimismo, se debe incluir espacios relevantes en donde el target interactúe, buscando influenciar opiniones y opinadores, mejorando resultados de los motores de búsqueda, y analizando la información que estos medios provean para optimizar el rendimiento de las acciones tomadas.

\section{Mix de marketing}

De acuerdo a Kotler y Armstrong la mercadotecnia se define como "el conjunto de herramientas tácticas controlables de mercadotecnia que la empresa combina para producir una respuesta deseada en el mercado meta. La mezcla de mercadotecnia incluye todo lo que la empresa puede hacer para influir en la demanda de su producto (2007: 154)"

El Diccionario de Términos de Marketing (2019) de la American Marketing Association, define a la mezcla de mercadotecnia como aquellas "variables controlables que una empresa utiliza para alcanzar el nivel deseado de ventas en el mercado meta. En síntesis, la mezcla de mercadotecnia es un conjunto de variables o herramientas controlables que se combinan para lograr un determinado resultado en el mercado meta, como influir positivamente en la demanda, generar ventas, entre otros."

A mediados de la década de los '60, Jerome McCarthy (premio Trailblazer de la American Marketing Association) introdujo el concepto de las 4 P's, que hoy por hoy, se constituye en la clasificación más utilizada para estructurar las herramientas o variables de la mezcla de mercadotecnia (Baby \& Londoño, 2008).

Las 4 P's consisten en: Producto, Precio, Plaza (distribución) y Promoción. 
Producto: Es el conjunto de atributos tangibles o intangibles que la empresa ofrece al mercado meta.

Precio: Se entiende como la cantidad de dinero que los clientes tienen que pagar por un determinado producto o servicio. El precio representa la única variable de la mezcla de mercadotecnia que genera ingresos para la empresa, el resto de las variables generan egresos.

Plaza: También conocida como Posición o Distribución, incluye todas aquellas actividades de la empresa que ponen el producto a disposición del mercado meta.

Promoción: Abarca una serie de actividades cuyo objetivo es: informar, persuadir y recordar las características, ventajas y beneficios del producto

\section{Comercio electrónico E-Commerce}

El comercio electrónico es la compra y venta de productos o servicios a través de plataformas en línea que contribuyen a una red diversificada de consumidores y proveedores en diferentes partes del mundo.

Picazo, Ramírez y Luna (2014) definen el comercio electrónico (CE) como el proceso de automatizar la compra y venta de bienes y servicios, mediante la utilización de una aplicación informática para satisfacer las necesidades de los clientes y las empresas.

Asimismo, puede cambiar la manera de conducir los negocios. A pesar de su reciente auge, tiene el potencial para alterar radicalmente las actividades económicas y el ambiente social de las sociedades contemporáneas (Andrews, 2002). Afecta a sectores tan vastos como las telecomunicaciones, las finanzas y la industria detallista y mantiene promesas en áreas como la educación (Millán \& Camilo, 2015) la salud y el gobierno.

Wigand (1997) define e-Commerce como la aplicación de tecnología de información y comunicaciones a la cadena de valor desde su punto de origen hasta su punto final, sobre procesos conducidos electrónicamente y diseñados para el cumplimiento de objetivos del negocio. Estos procesos pueden ser parciales 0 completos y pueden abarcar transacciones negocio a negocio (B2B), así como negocio a consumidor(BTC) y consumidor a negocio(C2C).

Según Wigand (1997) B2B se refiere a la expresión business to business, es decir, de negocio a negocio y se relaciona principalmente con el comercio mayorista, aunque también puede referirse a prestación de servicios y consumo de contenidos. Por ejemplo, los portales tipo marketplace en donde unas empresas especifican sus demandas de productos o servicios y otras los ofertan o aquellos en los que una 
empresa no trata de comunicarse con su cliente final sino con distribuidores, partners, importadores u otro tipo de comprador profesional.

En los modelos de negocio B2B, se suele especificar el target a partir de las características de las empresas a las que queremos comunicar nuestra promoción:

- Actividad de la empresa.

- Situación geográfica de la empresa.

- Ámbito en el que desarrolla su actividad.

- Dimensión: trabajadores, red de distribución, facturación, ventas...

- Etc.

En los modelos B2B, debemos considera las motivaciones y objeciones de los distintos perfiles del target:

- Decisor

- Prescriptor

- Comprador

- Consumidor

B2C es un modelo de negocio de comercio directo. Aunque este formato de venta directa del productor al cliente ya existía con anterioridad, Wigand, hace mención de que la irrupción de Internet ha permitido facilitar aún más la venta por parte del propio productor, sin la presencia de intermediarios entre él y el cliente. Cuando nos referimos a B2C, hay cinco tipos diferentes de empresas:

- Vendedores directos

- Intermediarios en Línea que son una especie de "intermediario" que pone en contacto a compradores y vendedores. Sin embargo, no son dueños de los productos o servicios en sí mismos.

- Basados en Publicidad se refiere a las compañías que utilizan grandes volúmenes de tráfico web para vender anuncios en línea.

- Basado en la comunidad utilizan comunidades en línea de personas que comparten un interés común.

- Basados en Honorarios como lo hace netflix que cobra una comisión por medio de una suscripción para acceder a sus servicios.

El comercio electrónico ha traído consigo muchas oportunidades para los diferentes mercados, la distribución del producto o servicio puede ser mejorada, esto se da gracias al uso de la Web haciendo que las empresas y los clientes puedan interactuar directamente, eliminando, por lo tanto, a los intermediarios y haciendo que las entregas sean de manera inmediata.

Al mejorar la comunicación comercial, la empresa mantiene constantemente actualizado a sus clientes acerca de sus productos o servicios y da la posibilidad de que los clientes puedan acceder a información de la empresa las 24 horas del día, no interesando el lugar donde se encuentren. 
Con respecto del mercado interno de México se asegura que el comercio electrónico ha tenido un despunte como mecanismo utilizado para realizar actos de comercio (León, 2019). Las perspectivas económicas del comercio electrónico en México son alentadoras; sin embargo, existen factores que se requieren atender con el objetivo de continuar con esta tendencia positiva, de los cuales se destacan:

a) Accesibilidad a la internet, no toda la población cuenta con un fácil acceso debido a sus posibilidades económicas.

b) Seguridad, la seguridad es un factor importante al momento de realizar transacciones en línea, según menciona María Mercedes Albornoz en su artículo "La falta de confianza en el comercio electrónico", El comercio electrónico necesita un nivel elevado de confianza por parte de los sujetos involucrados para poder desarrollarse y continuar expandiéndose. Dicha confianza es susceptible de variar de un sitio web a otro y también de país en país, producto de los diferentes entornos culturales locales. Lo cierto es que cuanto mayor sea el grado de confianza, mayor será el aprovechamiento de las ventajas del comercio electrónico.

La American Marketing Association (AMA) asegura que diversos aspectos de la actividad comercial electrónica pueden ser objeto de desconfianza por parte de PyMEs y consumidores: la identidad de la contraparte y la autenticidad de sus manifestaciones de voluntad, la seguridad en la transmisión de sus datos personales y los relativos a sus tarjetas bancarias así como el uso que la otra parte de la transacción o terceros poco éticos puedan hacer de ellos, el riesgo de incumplimiento del vendedor o prestador de un servicio, la disponibilidad real de un tercero neutral que esté a su alcance para la solución efectiva de eventuales controversias.

c) Accesibilidad, implica que los sitios de visita sean aptos para el uso de cualquier persona, inclusive las que padecen alguna discapacidad.

d) Oferta y logística

e) Protección jurídica al ciber consumidor

Para poder controlar los aspectos mencionados con anterioridad en los incisos b y e, la Norma Mexicana NMX-COE-001-SCFI-2018 en dónde se estipulan las disposiciones a las que se sujetarán todas aquellas personas que ofrezcan, comercialicen o vendan bienes, productos o servicios (Martínez \& Pino, 2016). Las actividades a las que se refiere esta Norma Mexicana se interpretarán y aplicarán bajo los principios de neutralidad tecnológica, autonomía de la voluntad, y equivalencia funcional, contemplados en el artículo 89 del Código de Comercio.

\section{Plataformas de comercio electrónico.}

Los contenidos de este artículo están bajo una licencia de Creative Commons Atribución No Comercial - Sin Obra Deriva da 4.0 Internacional 
Según Cantón Araujo las plataformas de comercio electrónico son uno de los principales actores en la venta a través de Internet son las plataformas digitales para el comercio electrónico, las cuales básicamente son aplicaciones web desarrolladas específicamente para el comercio o venta a través de Internet (2014: 6).

Entre las principales características de las plataformas de comercio electrónico destacan:

- Catálogo de productos. Las plataformas suelen facilitar la creación, categorización y mantenimiento de los productos que se desean vender en la tienda.

- Personalización de la apariencia de la tienda, ya que no tendría ninguna ventaja de marketing que todas las tiendas fuesen iguales y no pudiesen diferenciarse.

- Soporte de transacciones bancarias como medio de pago en la tienda, este soporte puede ser a través de pasarelas de pago electrónico para realizar transacciones con tarjetas de crédito, mediante transferencia bancaria o interoperabilidad con otros sistemas como PayPal.

- Gestión de pedidos. Muchas aplicaciones para eCommerce aportan facilidades para el envío y seguimiento de las mercancías.

- Creación de informes y estadísticas sobre la tienda. Todas estas funcionalidades han de prestarse en un marco de seguridad adecuado a la gestión de transacciones y datos propios de una plataforma de comercio electrónico. Así, su diseño debe contemplar la capacidad para afrontar ataques y proteger frente a los mismos los datos tanto de la compañía como de los usuarios de la plataforma.

\section{Medios de pago}

El término dinero electrónico es utilizado en forma general para referirse a una amplia gama de mecanismos de pago utilizados en el comercio electrónico. Se define el dinero electrónico como aquellos productos que permiten al consumidor utilizar medios electrónicos para acceder a otros servicios convencionales de pago (Martínez et al, 2009).

En la actualidad no se ha adoptado formalmente en el ámbito internacional una terminología respecto del dinero electrónico. Aquellas más utilizadas son smartcards, e- money, digital cash, cybermoney, cybercurrency y cyberpayments. Muchas veces un mismo término puede tener sentidos diferentes, según el contexto y las circunstancias en que se utilice (Devoto, 1992). 
El comercio electrónico, si bien se emplea, no se ha visto como tal; esto debe a que se usaba a través de medios de transmisión como televisión o radio que daba como resultado, que la mayor parte de las compras reales tuviesen lugar fuera de la red. Con la aparición progresiva de nuevos medios de pago digitales se están viabilizando la existencia de transacciones comerciales realizadas completamente a través de Internet.

a) Tarjetas: Las tarjetas pueden ser de crédito o débito. La utilización de las tarjetas en el e-commerce, presenta ciertos inconvenientes que se relacionan, principalmente con los siguientes aspectos:

- Seguridad. La tarjeta como medio de pago, requiere la transmisión (Candelario \& Rodríguez, 2015) al vendedor del número de tarjeta de crédito o débito. Si el mensaje no está encriptado, se corre el riesgo de que la transmisión sea interceptada y que la información allí contenida sea utilizada por terceros.

- Costos de transacción. Los emisores de tarjetas de crédito o débito incurren en costos al proveer el sistema de pago, los cuales en general son trasladados a los vendedores. Ello torna inconveniente el sistema para compras de poco valor.

- Limitación en la cantidad de personas que tienen tarjeta. Utilizar la tarjeta como medio de pago en el Comercio Electrónico exige que, tanto el comprador como el vendedor deben estar adheridos al mismo sistema de tarjeta de crédito o débito, caso contrario no podrá ser utilizado este mecanismo.

Dos de las tarjetas más importantes del mundo (MasterCard y Visa) utilizan una norma en común en el Comercio Electrónico: SET (Secure Electronic Transaction). Esta tecnología intenta superar cinco grandes desafíos:

- "Garantizar reserva en la información de pedidos y pagos, que se logra por la encriptación de los mensajes.

- Asegurar la integridad de todos los datos transmitidos, a través de la firma digital.

- Verificar que el titular de la tarjeta sea usuario legítimo de una cuenta, mediante la utilización de la firma digital y los comprobantes del comerciante.

- Garantizar la autenticidad del comerciante para que pueda aceptar pagos con tarjetas bancarias a través de una institución financiera. Facilitar y alentar la interoperabilidad entre proveedores de redes y de software (Reibán, 2012)". 
b) Cheques digitales: Este sistema funciona como si los cheques fueran reales, con la salvedad de que el cliente utiliza una firma digital para firmar dicho cheque. Una vez firmado el cheque debe transmitirlo on-line, encriptado. Para utilizar este sistema de pago, el cliente debe tener una chequera electrónica. Es una tarjeta del mismo tamaño que una tarjeta de crédito o débito que contiene los datos del cliente y se pasa por un slot, que se debe conectar a la computadora.

c) Dinero electrónico: La expresión dinero electrónico, en general, se refiere a una variedad de sistemas propuestos, ensayados o en plena operación durante los últimos cinco años, destinados a facilitar los pagos en transacciones minoristas. En particular, se refiere a valor (García et al, 2020) pre-almacenado en tarjetas inteligentes, del tamaño de una tarjeta de crédito, o en el disco rígido de computadoras personales, mediante programas especiales. Dicho valor es luego susceptible de ser transferido a otras tarjetas o computadoras similares, a comerciantes unidos de la necesaria infraestructura para recibirlo (lectoras de tarjetas) o a través de Internet u otras redes.

\section{Conclusión}

Mientras que una pequeña tienda tradicional tal vez nunca tenga que enfrentarse a la posibilidad de cumplir con órdenes de otras naciones, una tienda en línea tiene el potencial para vender al mundo entero. Al negociar con personas de otros países la empresa debe tener en cuenta que los servicios postales no siempre son eficientes y rápidos con la entrega de paquetes. Las empresas de E-Commerce deben tener en cuenta también que los consumidores pueden manejar diferentes unidades de medida, unidades monetarias, y lenguaje.

El sitio de comercio electrónico tiene que ser capaz de trabajar en unidades métricas o imperiales. La capacidad de trabajar en diferentes monedas es una característica amigable con el cliente, permitiendo a los clientes cotizar adecuadamente los productos en su propia moneda. El sitio de comercio electrónico también debe ser capaz de bloquear ciertos países y simplemente rechazar pedidos de aquellos con altos índices de fraude.

\section{Bibliografía}

Baby M., Jaime, y Londoño J., Juan Gonzalo. (2008). Las P 's de Mercadeo. Algunas precisiones. Revista AD-minister, núm. 12, 149-161.

Candelario Samper, Juan José y Rodríguez Bolaño, Moisés. (2015). Seguridad informática en el siglo XX: una perspectiva jurídica tecnológica enfocada 
hacia las organizaciones nacionales y mundiales. Revista Publicaciones e Investigación, 9, 153-162.

Cantón Araujo, David. (2014). Guía básica de seguridad en Magento. España: Ministerios de Industria, Energía y Turismo.

Diario Oficial de la Federación. Código de Comercio.1889.

Devoto, Mauricio. (1992). La economía digital: el dinero electrónico y el lavado de dinero. Revista Informática y Derecho, 5, 469-502.

Diario Oficial de la Federación. (2018). Declaratoria de vigencia de la Norma mexicana NMX-COE-001-SCFI-2018. Comercio electrónico-disposiciones a las que se sujetarán aquellas personas que ofrezcan, comercialicen o vendan bienes, productos o servicios.

Diccionario de marketing, 2019. Definición de marketing. Recuperado de https://www.foromarketing.com/glosario-de-marketing/

Fischer, Laura y Espejo C. Jorge A. (2004). Mercadotecnia. México: McGraw-Hill.

García Cálcaneo, Cynthya Capella; Martínez Prats, Germán; Silva Hernández, Francisca \& Guzmán Fernández, Candelaria. (2020). Análisis a la propuesta de la aplicación del Impuesto al Valor Agregado al comercio electrónico en México. Revista Ciencias de la Documentación, 6(1), 79-92.

Kotler, P. y Armstrong, G. (2007). Marketing, versión para Latinoamérica. España: Pearson.

Kotler, Philip y Armstrong, Gary. (2003). Fundamentos de marketing. España: Pearson.

Leon Ale, Fran. (2019). Marketing Digital: estas son las funciones de la Mercadotecnia.

López, Luis Martínez; Mata Mata, Francisco y Rodríguez Domínguez, Rosa María. (2009). Sistemas de pago seguro. Seguridad en el comercio electrónico. Revista de Estudios Empresariales, 1, 63-76.

Martínez Prats, Germán, Álvarez García, Yasmín Isolda, Silva Hernández, Francisca \& Tagle Zamora, Daniel. (2020). Environmental Taxes. Its Influence on Solid Waste in Mexico. Journal of Environmental Management and Tourism, 11(3), 755-762.

Martínez, John y J. Pino, Francisco. (2016). Definición de un Modelo de Calidad de Servicios Soportado por Tecnologías de la Información (TI). Revista Publicaciones e Investigación, 10, 49-67. 
Millán Cifuentes, Andrés y Felipe González, Claudio Camilo. (2015). Marco de Referencia para la Gestión de TI Centrada en la Creación de Valor Compartido, Publicaciones e Investigación, 9, 163-176.

McCarthy, E. Jerome; D. Perreault, William. (1996). Marketing: planeación estratégica, de la teoría a la práctica. Santafé de Bogotá: McGraw-Hill.

Ochoa G, Nancy E., Cruz B, Ingrid M., E Gil, Celio; Silva Chaves, Cristian Camilo; Grajales B. Sara Ketherine; Vergara Vargas, Leidy Lorena; Perea Díaz, María Paula \& Páez, Alonso. (2015). Estrategias En La construcción De Un Prototipo Como Modelo Integral En La gestión Investigativa Orientado Hacia El Esquema De Negocio. Revista Publicaciones E Investigación, 9, $113-134$.

Picazo-Vela, Sergio; Ramírez Goñi, Priscila Nathalí \& Luna Reyes, Luis Felipe. (2014). Comercio electrónico y emprendimiento: un análisis aplicando la teoría del comportamiento planeado. RECAl: Revista de Estudios en Contaduría, Administración e Informática, 2(5), 1-20.

Reibán Morales, María Paola. (2012). Estudio, diseño e implementación de un sistema de comercio electrónico para la empresa Atenas tours operadora de turismo. Cuenca.

Roncancio, Enrico. Artesanía. 1999.

UNESCO. (2017). Artesanía y diseño. Construir la confianza - La artesanía, elemento del desarrollo.

W. Andrews. (2002). Comercio Electrónico: Estrategia real, beneficios reales. EUA: Gartner Group.

Wigand, R. T. (1997). Electronic data interchange: A transaction cost perspective. EDI Forum, 10(1).

Cómo citar este artículo:

Martínez Prats, G., Jiménez Cervantes, V. ., \& Silva Hernández, F. . (2021). Comercio electrónico como medio de estrategia para el impulso de productos artesanales . Revista De Investigación Académica Sin Frontera: División De Ciencias Económicas Y Sociales, (35). https://doi.org/10.46589/rdiasf.vi35.372

https://revistainvestigacionacademicasinfrontera.unison.mx/index.php/RDIASF/arti $\underline{\text { cle/view/372 }}$ 

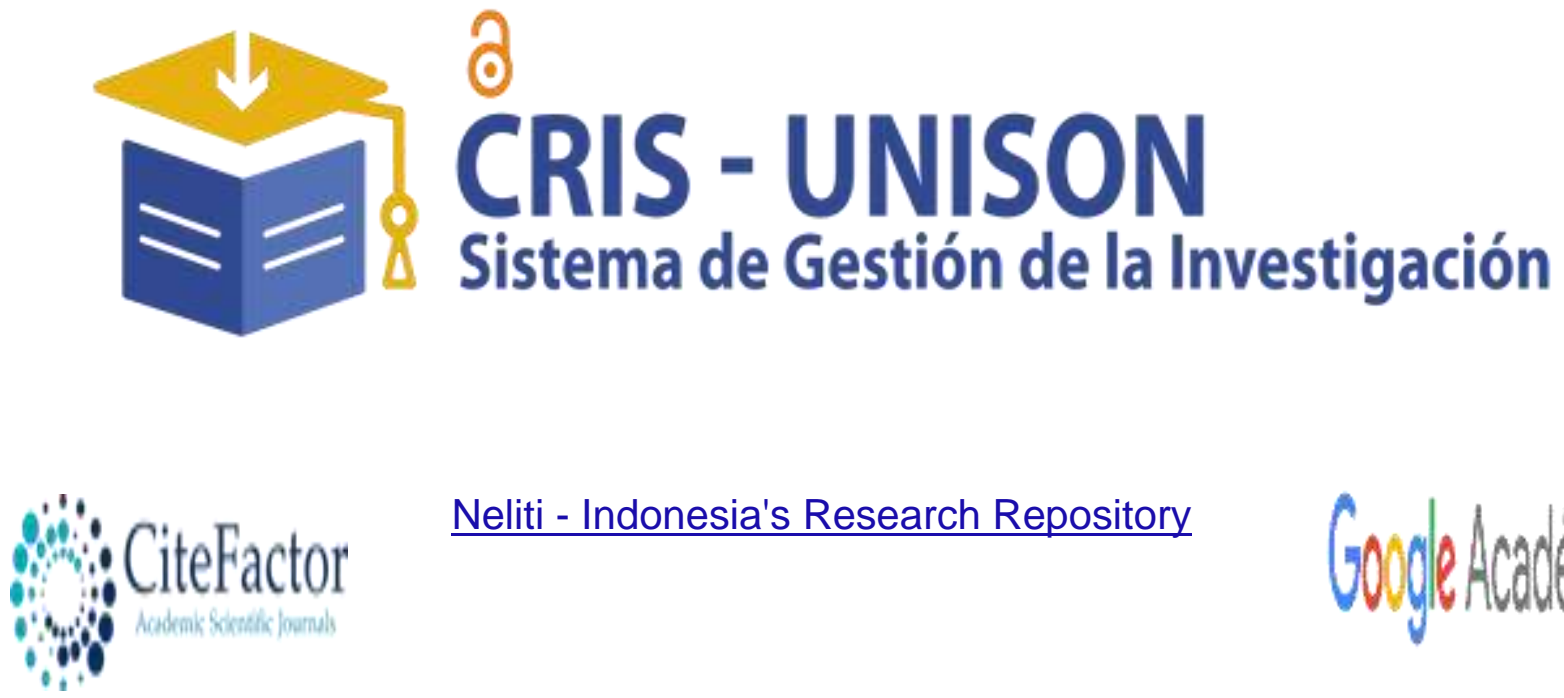

Neliti - Indonesia's Research Repository

DDialnet
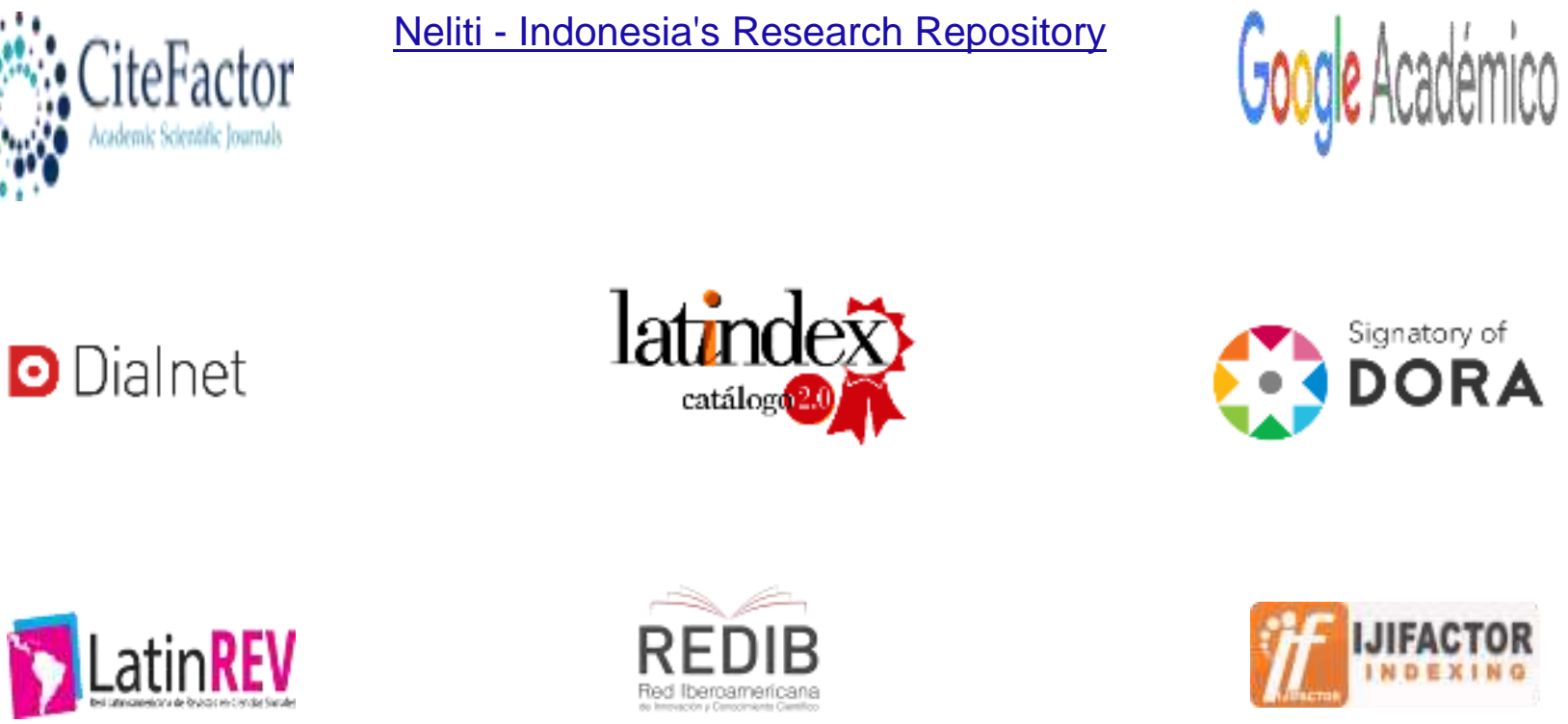

REDIB
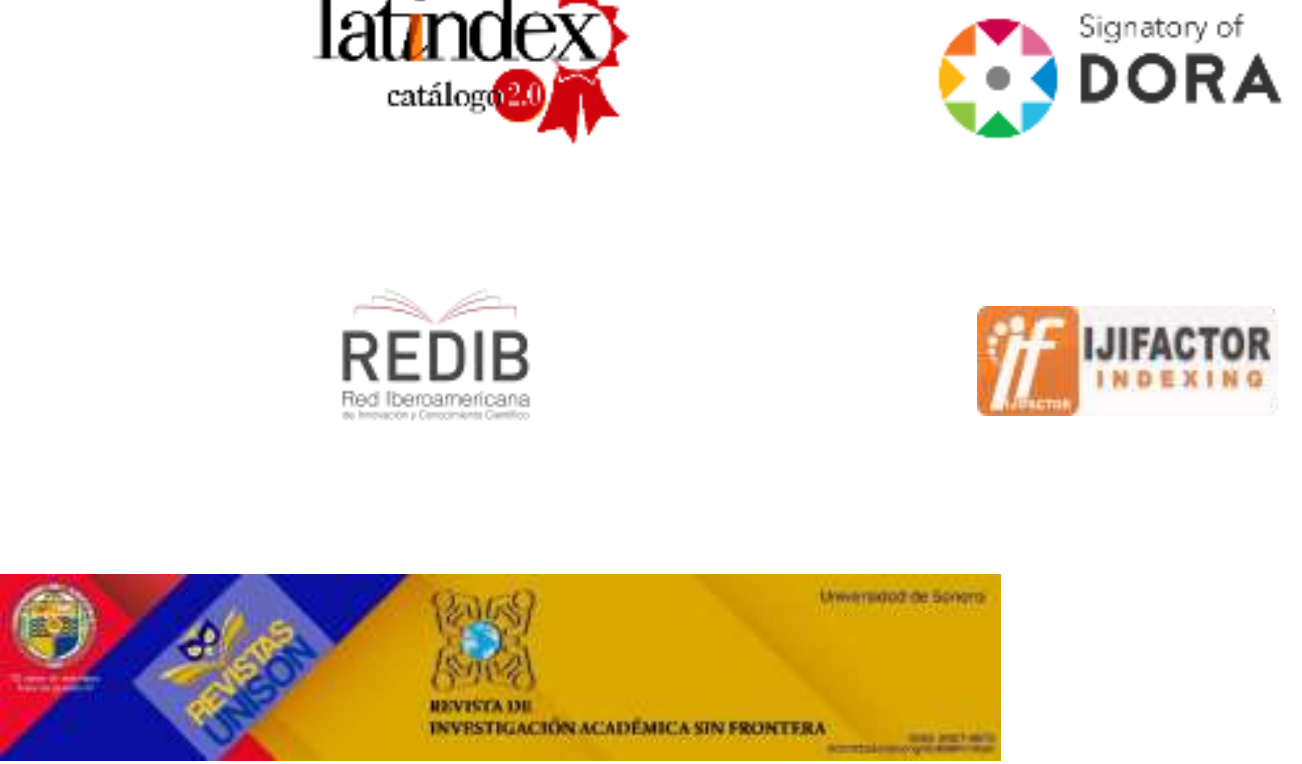